\title{
Prionacestus bipartitus N. GeN., N. SP. (Cestoda, Tetraphyllidea), Un Cas de néoténie
}

\author{
METE K.* \& EUZET L.*
}

\begin{abstract}
Summary : PRIONACESTUS BIPARTTTUS N. GEN., N. SP. (CESTODA, Tetraphyllidea) as the RESUlt OF a NeOTENY.

The Cestoda Tetraphyllidea Pelichnibothrium speciosum Monticelli, 1889 attribued to Yamaguti (1934) was found in typical host Prionace glauca (Selachii, Carcharhinidae). This parasite is not equivalent to the species of Monticelli. We propose to name it Prionacestus bipartitus n. gen., n. sp. The persistance of larval characters in the adult can considered as the result of a neoteny.
\end{abstract}

KEY WORDS : Prionacestus bipartitus, Tetraphyllidea, parasite, spiral intestine, Prionace glauca, neoteny.

\section{INTRODUCTION}

\section{A}

u cours d'une campagne de pêche aux grands poissons pélagiques dans le Sud Ouest de l'Océan Indien, l'un de nous (M. K.) a récolté chez quatre Prionace glauca (L., 1758) de nombreux individus d'un Cestode qui ressemble à celui décrit par Yamaguti (1934), chez le même hôte, sous le nom de Pelichnibothrium speciosum Monticelli, 1889. Le mélange, chez ce parasite, de caractères adultes et larvaires nous a amenés à revoir son attribution générique et spécifique, sa position systématique et à discuter de son originalité

\section{MATÉRIEL ET MÉTHODES}

T es parasites ont été récoltés dans la valvule spirale de Prionace glauca. Le plus rapidement possible après la capture du requin, sa cavité abdominale est ouverte par une incision médio-ventrale allant de la ceinture scapulaire à la région anale. L'appareil digestif ainsi mis à jour est détaché par deux incisions, une antérieure au niveau de l'œsophage et une postérieure au niveau du rectum. La valvule spirale est ensuite séparée de l'estomac un peu

\footnotetext{
* Station Méditerranéenne de l'Environnement Littoral, 1, quai de la Daurade, 34200 Sete.

Tél. : 04674633 74. - Fax : 0467460256.

Correspondance : L. Euzet.
}

\begin{abstract}
Résumé :
Le Cestode Tetraphyllidea attribué par Yamaguti (1934) à Pelichnibothrium speciosum Monticelli, 1889 est retrouvé chez I'hôte type Prionace glauca (Selachii, Carcharhinidae). Ce parasite ne correspondant pas à l'espèce de Monticelli, on propose de le nommer Prionacestus bipartitus n. gen., n. sp. La persistance chez l'adulte de caractères larvaires amène à considérer cette espèce comme résultant d'une néoténie.
\end{abstract}

MOTS CLÉS : Prionacestus bipartitus, Tetraphyllidea, parasite, valvule spirale Prionace glauca, néoténie.

en avant du pylore. Après incision longitudinale, la lame formant la valvule est déroulée et le contenu récupéré dans un bac contenant de l'eau de mer. Les Cestodes récoltés, maintenus dans l'eau de mer jusqu'à leur relaxation, sont fixés en les agitant dans de l'alcool à $70^{\circ}$. Certains individus ont été fixés aplatis entre deux lames. Après lavage à l'eau distillée, les parasites sont colorés avec le carmin acétique de Grenacher puis déshydratés, éclaircis dans un bain d'essence de girofle et montés entre lame et lamelle au baume du Canada. Les mensurations sont données en $\mu \mathrm{m}$ sauf avis contraire.

\section{RÉSULTATS}

PRIONACESTUS BIPARTITUS N. GEN., N. SP.

Hôte : Prionace glauca (L., 1758).

Habitat : valvule spirale.

Localité : Océan Indien $7,45^{\circ} \mathrm{S}$. $-52,16^{\circ} \mathrm{E}$. et $7,5^{\circ} \mathrm{S}$. $-53,06{ }^{\circ} \mathrm{E}$.

Matériel étudié : 10 individus colorés et montés.

Matériel déposé au Muséum National d'Histoire Naturelle (Paris) sous les numéros : 517 HF 20-21 et 22 CIX.

DESCRIPTION

On peut distinguer dans ces Cestodes deux parties : l'antérieure composée du scolex et du strobile et la postérieure représentée par une longue vésicule (fig. 1). Les deux parties, toujours unies sur le vivant, se séparent très souvent lors de la fixation. 


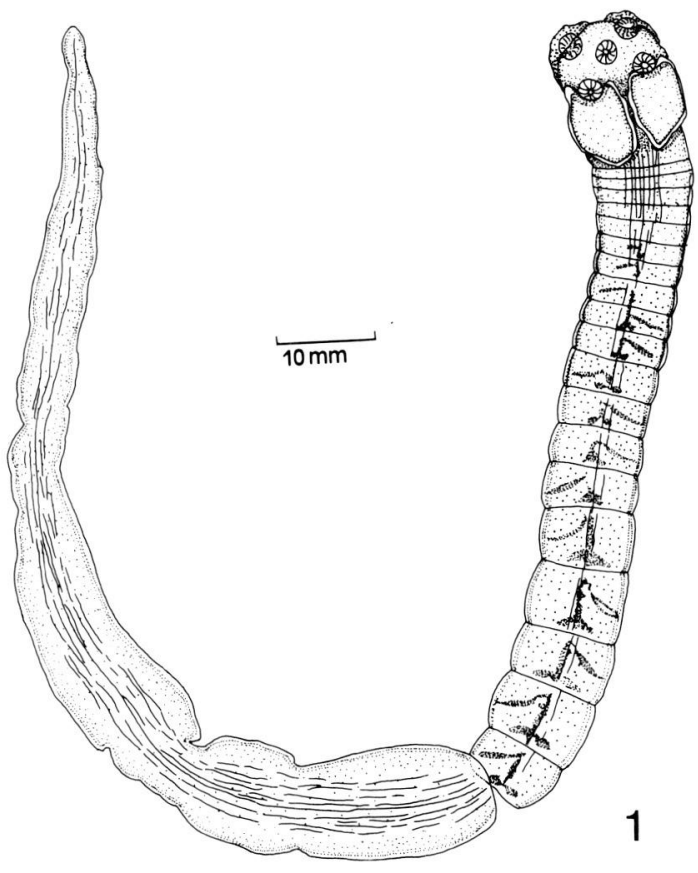

Fig. 1. - Prionacestus bipartitus n. gen., n. sp. Animal in toto (D'après Yamaguti,1934).

La taille des individus varie énormément, ainsi la partie antérieure mesure de 7 à $90 \mathrm{~mm}$ de longueur et de 1,5 à $3 \mathrm{~mm}$ de largeur au niveau des derniers segments du strobile et la vésicule postérieure de 11 à $110 \mathrm{~mm}$ de longueur et de 1,6 à $4 \mathrm{~mm}$ de largeur au point d'union avec le strobile marqué par une légère constriction. La vésicule s'élargit puis se rétrécit progressivement et se termine en pointe. Le cou, toujours plus large que le scolex, forme une gouttière largement ouverte ventralement. Comme Yamaguti l'a observé chez les proglottis détachés, les derniers segments du strobile, des individus fixés in toto, sont convexes dorsalement et concaves ventralement avec l'utérus formant une bosse dans la concavité.

Le scolex possède quatre bothridies sessiles (deux ventrales et deux dorsales) à bord simple légèrement plissé. Chacune possède à l'avant une ventouse accessoire de 250 à 300 de diamètre. Le scolex présente dans la région antérieure une avancée pyramidale à l'apex de laquelle s'ouvre une ventouse circulaire de 150 à 180 de diamètre (fig. 2). Les segments débutent presque immédiatement en arrière du scolex, d'abord plus larges que longs ( 1,5 à $3,4 \mathrm{~mm}$ sur 80 à 350 ), ils s'allongent graduellement très lentement. Les pores génitaux latéraux, situés à la moitié du segment, alternent irrégulièrement. Sur le plan médio-sagittal on peut observer un faisceau de fibres dans la moitié antérieure de certains proglottis aplatis.

Les testicules sont disposés en deux champs latéraux qui descendent presque jusqu'à la limite postérieure du segment. Le champ poral est interrompu au niveau

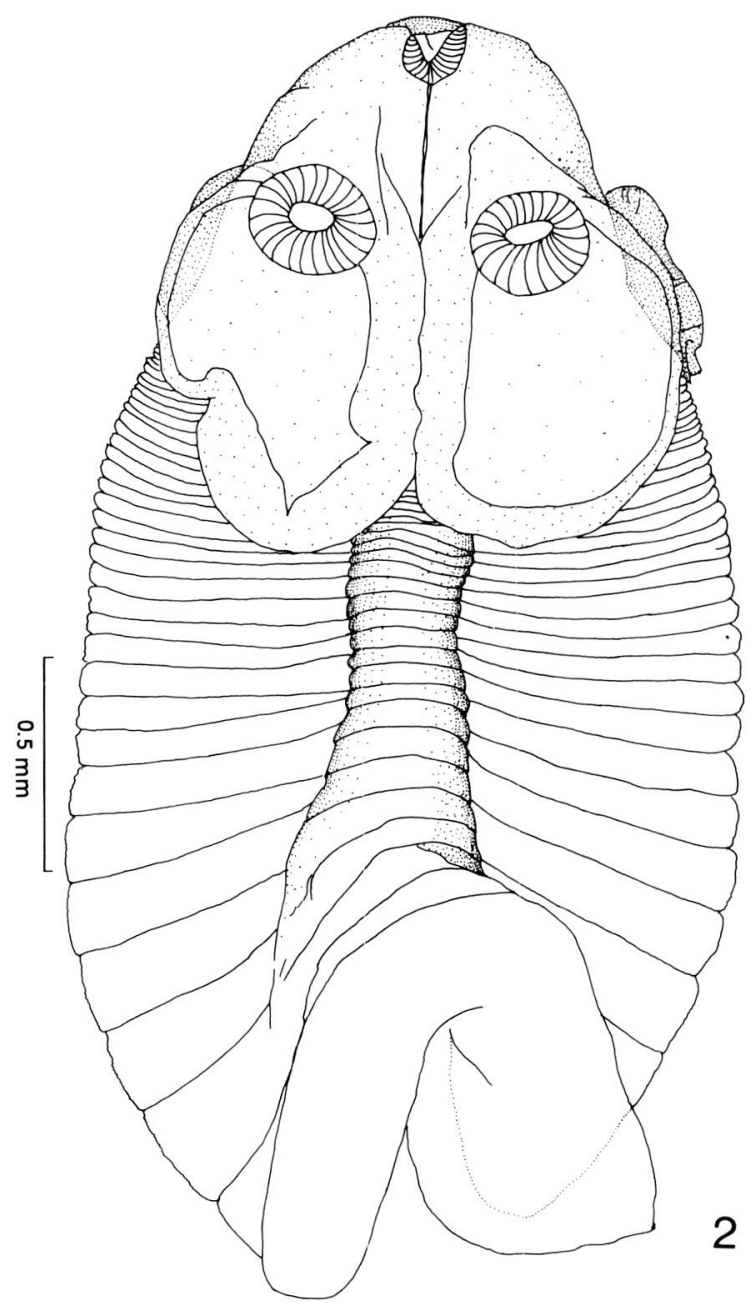

Fig. 2. - Prionacestus bipartitus n. gen., n. sp. Scolex avec l'élargissement antérieur du strobile formant une gouttière ventrale.

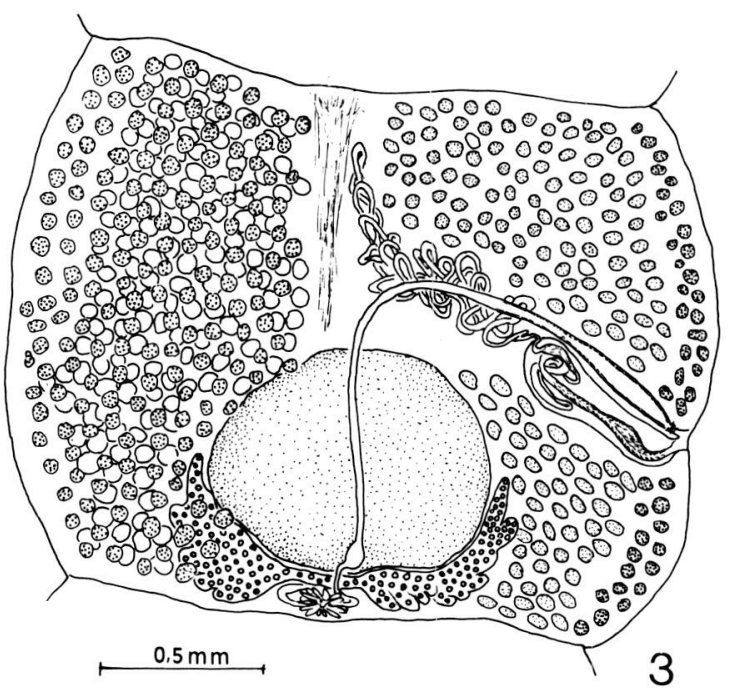

Fig. 3. - Prionacestus bipartitus n. gen., n. sp. Vue dorsale d'un proglottis gravide montrant le développement de l'utérus. Les follicules vitellins dorsaux ne sont représentés que sur la moitié gauche du segment. 
de la poche du cirre. Leur nombre est difficile à estimer à cause du développement des follicules vitellins. Chez quelques proglottis aplatis nous en avons compté de 260 à 300. Ce nombre est supérieur à ceux donnés par Yamaguti (1934), environ 200, et par Euzet (1959), 180 à 230. La poche du cirre, légèrement arquée vers l'avant, mesure 350 à 550 de longueur sur 100 à 120 de diamètre. Elle contient un long cirre armé de petites épines. Le canal déférent, empli de spermatozoïdes, forme des nombreuses circonvolutions dans la partie médiane du segment et aboutit à l'extrémité distale de la poche du cirre. A l'intérieur de celleci il se continue par un canal dont les circonvolutions sont parfois emplies de spermatozoïdes formant ainsi, avant le cirre proprement dit, une vésicule séminale interne.

L'ovaire, situé dans le quart postérieur du segment, est composé de deux masses latérales irrégulières unis par un isthme ovarien médian. L'ovaire est bilobé en coupe transversale. Le vagin s'ouvre antérieurement à la poche du cirre. Marqué à son début par un petit sphincter, il dessine une anse antérieurement puis se dirige sur le plan médian jusqu'à l'ovaire en avant duquel il s'élargit en un petit réceptacle séminal où nous avons observé des amas de spermatozoïdes. Les follicules vitellins forment deux larges bandes latérales recouvrant dorso-ventralement les testicules. Les glandes de Mehlis sont visibles comme une masse médiane juste en arrière de l'isthme ovarien. En avant de l'ovaire l'utérus forme, dans la moitié postérieure des segments, une poche médio-ventrale représentant le tiers environ de la largeur du segment (fig. 3). Les œufs utérins sphériques mesurent de 20 à 25 de diamètre.

La vésicule postérieure est constitué par un tissu parenchymateux très lâche avec, sur toute sa longueur, une bande médiane de fibres musculaires plus ou moins divergentes.

\section{DISCUSSION}

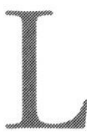

e Cestode Tetraphyllidea décrit ci-dessus correspond parfaitement à ceux que Yamaguti a récoltés en 1934 dans la valvule spirale de Prionace glauca sur la côte japonaise du Pacifique. Se basant sur la ressemblance morphologique du scolex, Yamaguti a nommé ce Cestode Pelichnibothrium speciosum, l'assimilant ainsi au grand plérocercoide signalé par Monticelli (1889) à Madère (Atlantique Est) dans l'intestin d'un Téléostéen pélagique Alopidosaurus ferox Lowe, 1833. Cependant, conscient des caractères très originaux du parasite de Prionace glauca, Yamaguti a proposé de le séparer dans la sous-famille nou- velle des Pelichnibothriinae au sein des Phyllobothriidae.

Southwell (1925) rejette le genre Pelichnibothrium parce qu'il est défini d'après la forme larvaire d'un adulte inconnu. Il propose cependant de conserver l'espèce signalée par Monticelli sous le nom de Phyllobothrium speciosum (Monticelli, 1889). Or, comme Zchokke et Heitz (1914) l'ont déjà remarqué à propos de Pelichnibothrium caudatum, les grandes larves à scolex de Tetraphyllidea trouvées dans l'intestin de Téléostéens représentent des étapes dans le développement de plérocercoides du type Scolex pleuronectis. Cette évolution a été observée par Euzet (1959) avec des larves de ce type trouvées dans l'intestin du Céphalopode Illex coindetii. Sur la seule morphologie des bothridies à bord non loculé et avec une ventouse accessoire, il est possible d'attribuer ces formes larvaires à un adulte appartenant à divers Phyllobothriinae : Phyllobothrium, Monorygma, Calyptrobothrium, Crossobothrium ou Clistobothrium.

Wardle et McLeod (1952) acceptent le genre Pelichnibothrium avec la définition de Yamaguti et considèrent que la plupart des grands plérocercoides qui ont été décrits chez les Téléostéens et les Céphalopodes appartiennent à ce genre. Cette position est aussi celle de Yamaguti qui, en 1959, reprend pour Pelichnibothrium sa diagnose de 1934. Mais en situant Pelichnibothrium parmi les Phyllobothriidae, il abandonne l'idée de créer pour ce seul genre une sousfamille particulière.

Euzet (1959) retrouve en Méditerranée, toujours dans la valvule spirale de Prionace glauca, un Cestode Tetraphyllidea qu'il assimile à $P$. speciosum, tel que l'a décrit par Yamaguti en 1934. L'utérus de cet individu étant toujours vide, il pense que ce parasite représente seulement une forme larvaire d'un Tetraphyllidea Phyllobothriidae. Cependant cette larve ne peut être assimilée à aucun des Tetraphyllidea connus chez Prionace glauca

Schmidt (1986) conserve le genre Pelichnibothrium et le situe parmi les Phyllobothriidae. Le seul critère distinctif retenu est la présence d'une ventouse à l'apex du scolex. La diagnose du genre, reprise d'après Yamaguti, comporte une erreur : le vagin est antérieur et non postérieur à la poche du cirre. En outre il n'y est pas fait mention de la présence d'une longue vésicule postérieure.

Pour les raisons citées plus haut, Euzet (1994) n'inclut pas Pelichnibothrium dans la clé de détermination des genres de Tetraphyllidea.

Yamaguti a assimilé le parasite de Prionace glauca à Pelichnibothrium speciosum sur un seul critère, la ressemblance du scolex. Il n'existe aucune preuve que le grand plérocercoide trouvé dans l'intestin d'Alopidosaurus ferox soit le stade larvaire du Cestode parasite 
de Prionace glauca tel qu'il a été observé au Japon, en Méditerranée et maintenant dans le sud-ouest de l'Océan Indien. Nous ne pouvons donc pas suivre Yamaguti lorsque il redéfinit le genre Pelichnibothrium Monticelli, 1889 d'après les individus récoltés dans la valvule spirale de Prionace glauca. Pelichnibothrium ne pourra être utilisé que pour la forme adulte correspondant au plérocercoide parasite d'Alopidosaurus ferox.

Ainsi le Cestode, parfaitement caractérisé, trouvé à plusieurs reprises dans la valvule spirale de Prionace glauca doit être renommé. Nous proposons Prionacestus bipartitus n. gen., n. sp. pour rappeler son hôte et sa biologie particulière.

\section{DiAgNose}

\section{Prionacestus n. gen.}

Synonyme : Pelichnibothrium sensu Yamaguti, 1934 nec Monticelli, 1889.

Tetraphyllidea, Phyllobothriidae. Phyllobothriinae néoténique avec un strobile suivi d'une longue vésicule. Scolex à quatre bothridies sessiles munies chacune d'une ventouse accessoire. Une ventouse apicale. Strobilisation débutant immédiatement en arrière du scolex. Pores génitaux latéraux alternant irrégulièrement. Testicules nombreux en deux champs médullaires latéraux. Vas deferens formant de nombreuses circonvolutions dans la région médiane des segments. Poche du cirre allongée. Cirre armé de petites épines. Ovaire postérieur formé de deux masses latérales unies par un isthme médian. Ovaire bilobé en coupe transversale. Vagin s'ouvrant en avant de la poche du cirre. Un sphincter vaginal. Follicules vitellins en deux larges bandes recouvrant dorsalement et ventralement les testicules. Utérus sacciforme médio-ventral. CEufs utérins sphériques. Adulte chez Prionace glauca (Selachii Carcharhiniformes).

\section{Espèce type : Prionacestus bipartitus n. sp.}

La découverte, chez quatre Prionace glauca, de nombreux Cestodes, adultes puisqu'ils possèdent des segments à utérus gravide, nous oblige à réfuter les hypothèses émises à savoir qu'ils représentent la postlarve d'un Tetraphyllidea encore inconnu.

Nous pensons que Prionacestus bipartitus doit être considéré comme une espèce néoténique puisqu'elle possède à la fois des caractères larvaires (scolex avec ventouse apicale et vésicule postérieure de plérocercoide) et des caractères d'adulte (segment à système génital fonctionnel et utérus gravide). Cependant même si nous avons observé des spermatozoïdes accumulés dans la partie terminale du vagin, nous ne connaissons pas la valeur ontogénique des oufs utérins puisque ceux que nous avons pu observer ne possédaient pas d'oncosphère entièrement formée. Il est cependant possible que le développement de ces oufs se déroule plus tardivement puisque Yamaguti a signalé des individus sans vésicule postérieure et des proglottis détachés.

\section{REMERCIEMENTS}

4 n nous recevant dans son laboratoire M. F. Rene, Directeur de la délégation de l'IFREMER à la Réunion, nous a associé aux recherches entreprises sur la biologie de l'espadon dans le sud-ouest de l'Océan Indien. Nous le remercions ainsi que tous les membres de son équipe. Nous remercions l'armement SATMER qui a permis notre embarquement à bord du palangrier "l'Amaryllis ". Nous adressons nos sincères remerciements à tous les membres de son équipage pour leur amical accueil et pour leur aide lors de nos récoltes.

\section{BIBLIOGRAPHIE}

EuzET L. Recherche sur les cestodes tétraphyllides des sélaciens des côtes de France. Thèse présentée à la Faculté des Sciences de Montpellier (1956). Causse-Graille \& Castelnau imprimeurs, 1959, $263 \mathrm{p}$

EuzET L. Tetraphyllidea. In : Key to the Parasites of Vertebrates. Khalil, Jones \& Bray (eds). Published by International Institute of Parasitology, CAB International, 1994, $751 \mathrm{p}$.

Monticell F.S. Notes on some Entozoa in the collection of British Museum. Proceedings of the Zoological Society, London, 1889, 321-325.

SCHMIDT G.D. CRC Handbook of Tapeworm Identification Boca Raton Florida, USA, CRC Pres, Inc., 1986, 675 p.

SouTHwell T. A monograph on the Tetraphyllidea with notes on related Cestodes. Memoirs of the Liverpool School of Tropical Medecine, 1925, New series n², 363 p.

WARDLE R.A. \& Mc LEOD J.A. The zoology tapeworms. University of Minnesota Press, Minneapolis USA, 1952, XXIV + $780 \mathrm{p}$.

Yamaguti S. Studies on the Helminth Fauna of Japan. Part. 4. Cestodes of Fishes. Japanese Journal of Zoology, 1934, 6, 1-112.

Zschokke F. \& HeITz A. Entoparasiten aus Salmoniden von Kamtschatka. Revue Suisse de Zoologie, 1914, 22, 205-217.

Reçu le 20 mai 1996 Accepté le 8 août 1996 